\title{
Research On The Inherent Characteristics Of The Wind Turbine Tower Based On DQM
}

\author{
Zhiqiang $\mathrm{Xu}$ \\ Department of Physics and Technology, Kunming University, Kunming 650214, China \\ 2793436659@qq.com
}

Keywords: The Wind Turbine; The Inherent Characteristics; Differential Quadrature Method; Vibration Characteristics

\begin{abstract}
DQM is widely used to solve common boundary conditions of a variety of structures, but rarely applied to the complex boundary conditions corresponding to the actual projects. Most of the time, what is obtained is the dimensionless frequency, which can not be applied directly to the general engineering design. In this paper, the common towering structure engineering is studied, which is equivalent to a variable cross-section beam mechanical model. DQM is adopted to find its natural frequency and do comparative analyses. Finally the inherent characteristics of structural variation under various conditions are summed up in order to provide a theoretical reference for issues related to projects. The consistency of the theoretical analysis and numerical solution suggests that DQM works feasibly in engineering calculation for this kind of model.
\end{abstract}

\section{Introduction}

Multi-body dynamics is an important tool for studying the dynamic behavior of complex mechanical system, which has developed in recent years. It establishes second order partial differential equations of characterization system dynamical behavior based on the principle of conservation of energy. It's not easy for this kind of partial differential equations to obtain the analytical solution Therefore, numerical algorithm - differential Quadrature method is utilized. Thin-walled plate theory is the theory which aims at the mechanical properties and characteristics of thin-walled structures of the objects and shows the internal deformation, the displacement as well as strain relationships of the thin-walled structures. Since the structure of the wind turbines studied in this paper is complex and special, its system dynamics modeling and analysis is required to base on the above theoretical methods. This paper briefly describes the related theoretical basis. What's more, the load and characteristics of wind turbines are introduced.

\section{Simplifying The Load Of The Wind Turbine}

Combined with the operating environment of the wind turbine, the following loads can be seen in the process of the operation of the wind turbine: the wind resistance of the wind and the nacelle, the wind resistance of the tower, the nacelle weight of the wind turbine and its bending moment generated by mass eccentricity as well as the driving torque while working, shown as Figure1.

The parameters in the figure are expressed as follows:

(1) the horizontal thrust of the wind wheel

$$
F_{x}=\frac{4}{9} \rho \pi R^{2} v^{2}
$$

$F_{x}$ - the horizontal thrust of the wind wheel, unit: $\mathrm{N}$

$\rho$ — the air density, unit: $\mathrm{kg} / \mathrm{s}^{3}$

$R$ - the radius of the wind wheel unit: $\mathrm{m}$

$v$ - the wind velocity, unit: $\mathrm{m} / \mathrm{s}$

(2) the weight of the wind turbine and the nacelle $G=m g$

$m$-The total mass of the rotor and the nacelle, unit: $\mathrm{kg}$

$g$ - the acceleration of gravity, unit: $\mathrm{m} / \mathrm{s}^{2}$ 


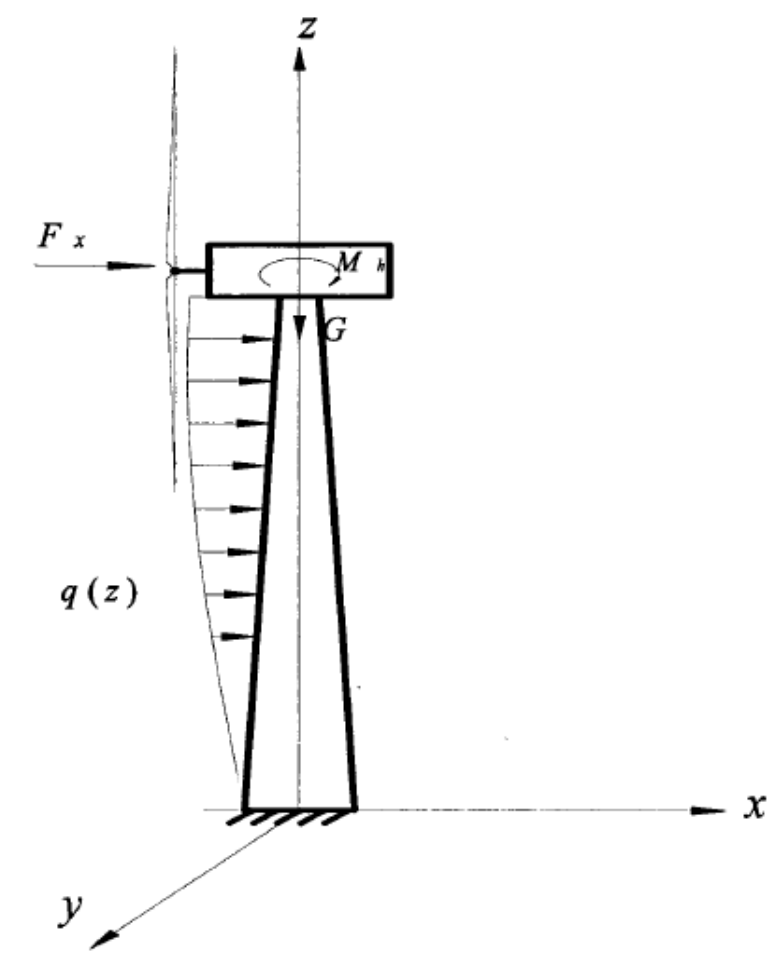

Fig 1. the force diagram of the wind turbine

(3) $q(z)$ the wind pressure along the height of the tower, unit: $\mathrm{Pa}$

(4) the bending moment generated by the thrust and the weight of the eccentric of the wind turbine nacelle

$$
M_{h}=F_{x} h_{1}+G h_{2}
$$

$M_{h}$ - the bending moment generated by the thrust and the weight of the eccentric of the wind turbine nacelle

$h_{1}$ - the height from the main wheelbase of the wind turbine tower to the tip

$h_{2}$ - the eccentric distance of the wind turbine nacelle system quality relative to the tower axis

(5) $M_{n}$-the torque of the wind wheel generating electric power and the moment of the friction generated by driving systems. When combined with the above stress analysis, $q(z)$ is ignored. When the wind turbine vibrates, the exciting force in $X$ direction is $F_{x}=\frac{4}{9} \rho \pi R^{2} v^{2}$.

Excitation torque is: $M_{h}=F_{x} h_{1}+G h_{2}$. Since the conservative force does not affect the structure of the inherent characteristics of free vibration, $G h_{2}$ can be ignored. Namely, the exciting moment can be seen as:

$$
M_{h}=F_{x} h_{1}
$$

\section{The Vibration Characteristics Of The Tower Beam Model By DQM}

Differential Quadrature Method[1,2] (abbreviated DQM) has developed rapidly in recent years, whose basic principle is to find the weight sum of all the function values of discrete points in the physical domain for a certain function. Then be close to the partial derivative or the integration at a discrete point of the function. Bellman and Casti [2] First used DQM to solve ordinary differential equations and boundary value. Bert and Malik used DQM to analyze the vibration of beams, plates, and other cylindrical shell structure [3, 4]. This is the beginning of DQM application in the field of mechanics. In Document [5], DQM is adopted to analyze the large deformation of the pile for in the elastic or elastoplastic soil. In Document [6], it is used to perform numerical calculations to axially moving viscoelastic and Beam with Nonlinear forced vibration. As an efficient numerical calculation method, differential quadrature method has been widely used in solid mechanics, fluid 
dynamics and other engineering disciplines

Currently, the error analysis and the error estimation of DQM as well as numerical stability are important issues worthy of study [7]. Because of that, the concept of shape function is introduced. Meanwhile, a mechanical model, the vibration control equation and the numerical result are connected. The error in the solution process, the region of convergence and numerical results of DQM are demonstrated. The impact of the change in the length of the beam on the natural frequency is examined. The local stiffness of the variable cross-section beam and the influence of the mass distribution on its binding modal shape are studied. Therefore, the parameters of the variable cross-section the girder cross-section section has the characteristics impact on the fixed beam. These results will provide a theoretical reference for the engineering design and troubleshooting.

The cone-type large tower is usually used in modern large-scaled wind turbine. According to its geometrical characteristics and mechanical characteristics, it can be simplified into a set of bending and axial compression deformation and torsional deformation as one of the beam's complex issues to. But because of its thin-walled structure, it will be a great error to solve the result. For this model, a beam shaping $1.5 \mathrm{MW}$ wind turbine tower dynamic characteristics is analyzed. Three models will be built, which are shown in Figure 2:

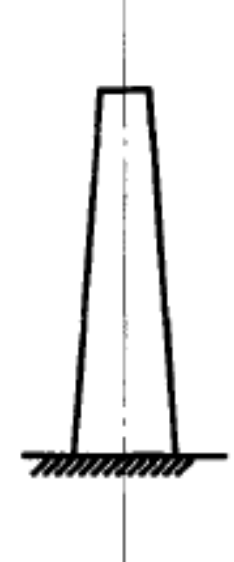

Mode1 1

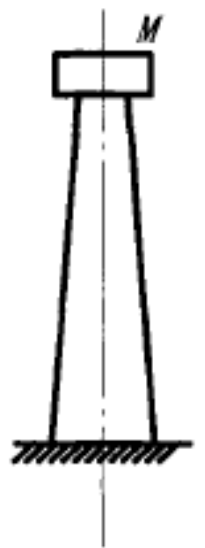

Model 2

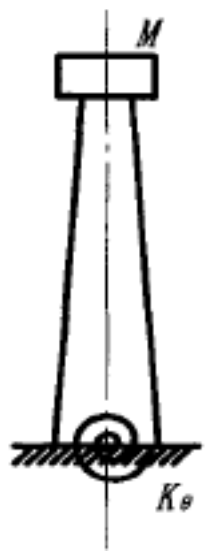

Model 3

Fig 2. the model Schematic of the wind turbine tower

Model 1: tower simplified to variable section tapered cylindrical cantilever;

Model 2: the inertia shear produced by the wind wheel and the nacelle mass in the tower at the top of Model 1;

Model 3:considering the rotational stiffness based on Model $2 K_{\theta}$.

The natural frequency is shown in Table 1

Table $1 \mathrm{~N}=35$ the natural frequency corresponding to three models of the wind turbine/ $\mathrm{Hz}$

\begin{tabular}{|c|c|c|c|}
\hline Frequency & Model l & Model 2 & Model 3 \\
\hline 1 & $5.2585 \mathrm{E}+00$ & $2.48320 \mathrm{E}+00$ & $1.2280 \mathrm{e}+000$ \\
\hline 2 & $2.6219 \mathrm{E}+01$ & $1.89080 \mathrm{E}+0 \mathrm{l}$ & $2.7616 \mathrm{e}+000$ \\
\hline 3 & $6.8620 \mathrm{E}+01$ & $5.58680 \mathrm{E}+01$ & $6.3688 \mathrm{e}+000$ \\
\hline 4 & $1.3192 \mathrm{E}+02$ & $1.13890 \mathrm{E}+02$ & $9.6705 \mathrm{e}+000$ \\
\hline 5 & $2.163 \mathrm{E}+02$ & $1.92980 \mathrm{E}+02$ & $1.2940 \mathrm{e}+00 \mathrm{l}$ \\
\hline
\end{tabular}

\section{Conclusion}

For the beam model of the wind turbine tower, when the inertia force produced by the nacelle mass of the wind turbine and the basic rotational stiffness is considered, there will be a clear decrease of natural frequencies when compared with a simple cantilever tower. It shows that the Inertia Shear generated by the mass of the wheel -nacelle system and the basic stiffness has a very significant impact on the inherent characteristics of the wind turbine, which should be fully considered. Therefore, the mass configuration of the head and foundation stiffness can be adjusted to change its 
inherent properties, which is better to keep away from the operating frequency. From Document[8], the basic frequency model is $2.0363 \mathrm{~Hz}$. The data in this paper show the frequency values of the wind turbine getting from the beam model. The assumption of the beam has determined that its stiffness value is greater than the actual value of the stiffness when it deal with the similar thin-walled beams hollow structure, resulting in significant increase in natural frequency.

\section{References}

[1]Bellman R E,Kashef B G'Casti J.Differential quadrature: a technique for the rapid solution of nonlinear partial differential equations[J].J Comput Phys,1 972,1 0:40—52.

[2]Bellman R E,Casti J.Differential quadrature and long-term integration[J].J Math Anal Appl,1971,34:235-238.

[3]Bert C W,Malik M.Differential quadrature method in computational mechanics:A review[J].Appl Mech Rev,1 996,49:1.27.

[4]Bert C W,Malik M.Free vibration analysis of thin cylindrical shells by the differential quadrature method[J]. Pressure Vessel Tech,1 996,11 8:1.1 22008,25(3):398.405.

[5] Yujia Hu, Yuanyuan Zhu, Changjun Cheng. Large deformation of the elastic or elastoplastic analysis of the soil in the pile [J]. Journal of Applied Mechanics, 2008,25 (3): 398.40

[6] Hu Ding, Liqun Chen. Axially moving beams for lateral vibration of multiscale analysis and DQM verification [J]. Vibration Engineering News, 2009,22 (3): 298.305

[7] Changjun Cheng, Zhengyou Zhu. Differential Quadrature Method and Its New Progress in mechanics applications [J]. Shanghai University (Natural Science), 2009,15 (6): 551.560

[8] Zhenzhou Zhao, Yuan Zheng, Yuqin Gao. Wind Turbine Principles and Applications [M]. Beijing: China Water Conservancy and Hydropower Publishing Society, 2011. 